Revista de Psicología de la PUCP. Vol. XXIII, 2, 2005

\title{
Comportamiento antisocial durante la adolescencia: teoría, investigación y programas de prevención
}

\author{
Dora Herrera Paredes ${ }^{1}$ \\ Pontificia Universidad Católica del Perú \\ Hugo Morales Córdova ${ }^{2}$ \\ Universidad Nacional Mayor de San Marcos
}

La multicausalidad del comportamiento antisocial durante la adolescencia parece no sólo obedecer a la combinación de múltiples factores de riesgo ubicados en diferentes niveles del desarrollo humano, sino también a procesos históricos y culturales que afectan de manera diferenciada a varias generaciones de jóvenes desde su temprana infancia. Este artículo revisa las principales teorías explicativas del comportamiento antisocial durante la adolescencia, enfatizando en la teoría neuropsicológica de la Taxonomía del Desarrollo de la Conducta Antisocial propuesta por Terrie E. Moffitt (1993, 1994, 1996, 2003). Asimismo, se mencionan algunos estudios realizados que confirman la validez transcultural del modelo teórico de Moffitt y sus contribuciones para el diseño de programas de prevención del delito entre población adolescente y juvenil en nuestro medio.

Palabras clave: adolescencia, conducta antisocial, desarrollo, prevención.

\section{Antisocial behavior during adolescence: theory, research and prevention programs}

The existence of several causes of antisocial behavior during adolescence seems to respond, not only to the combination of many risk factors within different levels of human development, but also to cultural and historical processes affecting, in many ways, several generations since their early childhood. This paper revises the main explicative theories about antisocial behavior during adolescence and highlights the theory of the Neuropsychological Taxonomy of the Antisocial Behavior proposed by Terrie E. Moffitt (1993, 1994, 1996, 2003). Moreover, some studies are mentioned due to the fact that they confirm the cross-cultural validity of Moffitt's theorical model and its contributions to the design of prevention programs against delinquency for youngsters and adolescents in our context.

Keywords: Adolescence, antisocial behavior, development, prevention.

Profesora Auxiliar del Departamento de Psicología de la PUCP y Profesora Asociada de la Facultad de Psicología de la Universidad de Lima. Doctora en Psicología por la Katholieke Universiteit Leuven, Bélgica, con estudios de post grado en Psicología y Antropología en Johns Hopkins University y en la PUCP. Licenciada en Psicología por la UNMSM. Consultora externa de la Organización Internacional del Trabajo. Correo electrónico: diherrer@pucp.edu.pe 

En los últimos 30 años, el estudio científico de la delincuencia adolescente y juvenil ha cobrado especial relevancia en la implementación de políticas públicas de prevención de la violencia a nivel mundial (Lahey, Moffitt \& Caspi, 2003). La prevención y rehabilitación de la delincuencia han sido favorables cuando se han realizado intervenciones "basadas en evidencia"; pero aún es necesario contrastar empíricamente estos resultados en contextos socioculturales distintos (Barletta \& Morales, 2004).

En el Perú, la tasa de violencia criminal expresada en delincuencia juvenil y sus efectos se ha incrementado durante los últimos años. Sólo en Lima, la capital del país, existe alrededor de 12,795 pandilleros (88\% de éstos tiene entre 12 y 24 años de edad) agrupados en más de 390 pandillas juveniles (DIRFAPACI, 2004). Los efectos de las acciones organizadas por pandilleros son muchas veces devastadores. Este es un fenómeno preocupante si consideramos que en la estructura poblacional peruana, la población comprendida entre los 15 y 24 años de edad representa el $19.4 \%$ del total poblacional estimado (INEI, 1998, 2004), mientras que en la capital el grupo de edad comprendido entre los 13 y 20 años representa el $16.4 \%$ del total poblacional capitalino estimado (APOYO Opinión y Mercado, 2003).

La participación de un elevado porcentaje de adolescentes varones en actos delictivos representa una importante amenaza para

2 Profesor de la Facultad de Psicología de la UNMSM y de la Escuela de Educación de la Universidad Peruana Unión. Licenciado en Educación por la UNMSM y Bachiller en Psicología por la PUCP. Coordinador de Proyectos de Instituto APOYO e investigador en Políticas Públicas de Desarrollo, Educación, Salud y Justicia Juvenil. Correo electrónico: morales.h@pucp.edu.pe 
el desarrollo humano y el crecimiento económico de una nación (OMS, 2003). De acuerdo a un estudio de la Defensoría del Pueblo (2000), en 1998 la mayoría de los adolescentes en conflicto con la Ley Penal bajo tutela Judicial (85\%) atraviesa sin éxito por los procesos de educación formal (CEAPAZ, 2000). Esto ocurre especialmente en aquellos que provienen de hogares desestructurados, incompletos y disfuncionales (62\%).

En aquellos contextos familiares, los adolescentes presentan desventajas individuales y cuidados negligentes a lo largo de su desarrollo; debido a ello, se involucran en actividades marginales y de alto riesgo psicosocial (Wright, Caspi, Moffitt \& Silva, 1999a, 1999b, 2001).

Sin embargo, es importante precisar que no todos los adolescentes que han cometido alguna infracción a la Ley persisten en este comportamiento a lo largo de su vida; ello ocurre pese al riesgo de los efectos de sus entornos criminógenos (Farrington, Jollife, Loeber, Stouthamer-Loeber \& Kalb, 2001; Seydlitz \& Jenkins, 1998). Aunque, ciertamente, existirá un pequeño grupo de adolescentes cuya conducta antisocial persistente los expone a desarrollar el Trastorno Antisocial de la Personalidad (APA, 1994).

Con fines preventivos y de rehabilitación, la identificación temprana de aquellas características individuales y sociales que discriminan entre adolescentes que persisten y no persisten en el comportamiento antisocial delictivo durante el curso de su vida, constituye una estrategia importante para el diseño de políticas sociales eficaces en el ámbito de la delincuencia juvenil (Kazdin \& Buela-Casal, 2001; Lerner \& Galambos, 1998). 


\section{Epidemiología de la delincuencia juvenil}

La delincuencia adolescente y juvenil, como forma de violencia criminal, es una de las formas de violencia más visible en una sociedad (Erikson, 1980; Garrido, 1997). Permanentemente, los medios de comunicación masiva informan sobre la actuación de las pandillas en las escuelas y en las calles (Seydlitz \& Jenkins, 1998). Estadísticas provenientes de los cinco continentes (ver Figura 1) advierten que tanto adolescentes como adultos jóvenes son las principales víctimas y perpetradores de este tipo de violencia (Farrington, 1983; OMS, 2003).

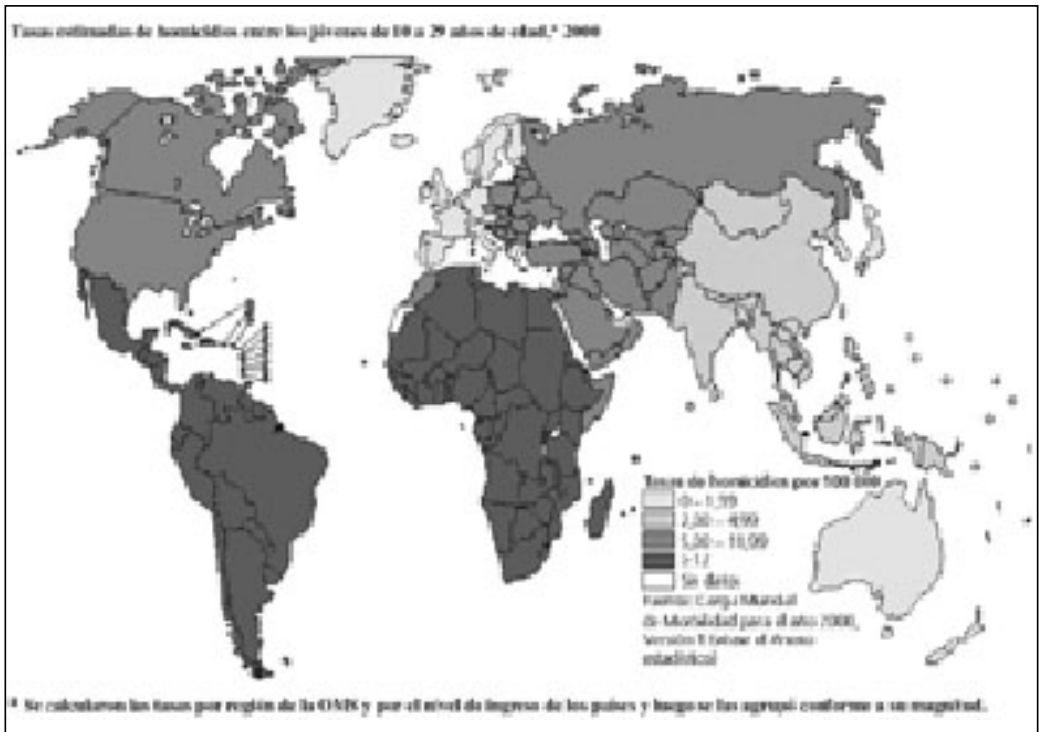

Figura 1. Tomada de Informe Mundial sobre la Violencia y la Salud, con autorización de la OMS (2003).

Los crímenes u homicidios, así como los delitos bajo la forma de agresiones no mortales que involucran a adolescentes y 
jóvenes (ver Figuras 2 y 3), incrementan enormemente la carga mundial de muertes prematuras, años de vida no productivos, lesiones y discapacidad (OMS, 2003).
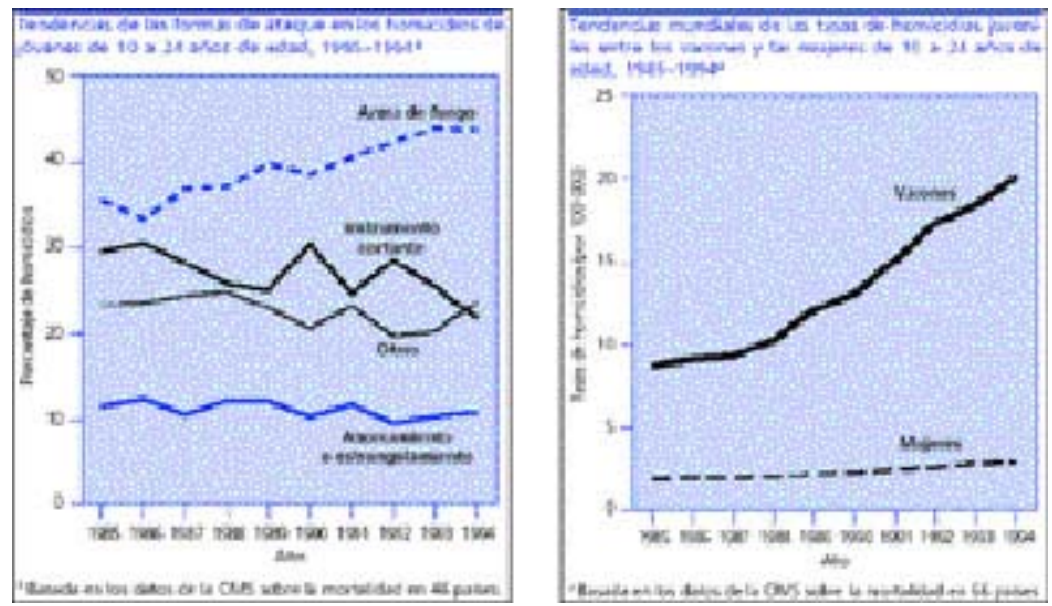

Figuras 2 y 3. Tomadas de Informe Mundial sobre la Violencia y la Salud, con autorización de la OMS (2003).

La violencia adolescente y juvenil daña profundamente no sólo a las víctimas, sino también a sus familias, amigos y comunidad (Lynam, Caspi, Moffitt, Wikstrom, Loeber \& Novak, 2000; OMS, 2003). Sus efectos pueden apreciarse no sólo en los casos de muerte, enfermedad y discapacidad de sus víctimas directas, sino también en la calidad de vida de los habitantes de una comunidad (víctimas indirectas) y el crecimiento económico de una nación (OMS, 2003). La violencia que afecta a los adolescentes y jóvenes y los hace delincuentes, incrementa enormemente los costos de los servicios de salud y asistencia social, reduce la productividad, disminuye el valor de la propiedad, desorganiza una serie de servicios esenciales y, en general, deteriora la estructura de una sociedad (Pattishall, 1994). 
En el Perú, hasta noviembre de 2004 la población total de adolescentes en conflicto con la Ley Penal atendidos por el Sistema de Reinserción Social del Adolescente Infractor del Poder Judicial representaba 1,411 personas $(65,9 \%$ adolescentes bajo sistema cerrado y $34,1 \%$ en sistema abierto), entre los cuales $38 \%$ se encuentra en condición de procesado mientras $62 \%$ se encuentra en situación de sentenciado (Gerencia de Centros Juveniles del Poder Judicial, 2005).

Según la Defensoría del Pueblo del Perú (2000), desde 1997 el número de adolescentes privados de libertad en los Centros Juveniles se ha incrementado en $90 \%{ }^{3}$, siendo las infracciones más frecuentes las referidas a delitos contra el patrimonio (46.4\%), contra la libertad sexual $(19.4 \%)$ y contra la vida, el cuerpo y la salud $(14.1 \%)$.

En 1998, con el apoyo del Instituto Latinoamericano de las Naciones Unidas para la Prevención del Delito y el Tratamiento del Delincuente (ILANUD), la Defensoría del Pueblo entrevistó a 467 adolescentes (438 hombres y 29 mujeres) internos bajo cargos penales en los Centros Juveniles de todo el país, con la finalidad de realizar un perfil personal, social, económico y jurídico del adolescente infractor peruano. Los resultados de este estudio señalan que el $85 \%$ de los adolescentes vive en circunstancias personales, sociales y económicas difíciles, y proceden de sectores de la población que viven en extrema pobreza. Asimismo, padecen de graves deficiencias en el plano educativo y tienen una baja o nula instrucción formal, siendo los índices de retraso y deserción escolar muy elevados (Defensoría del Pueblo, 2000).

3 En diciembre de 1997 eran 467, en febrero de 1999 eran 636, en agosto del 2000 eran 879 y en setiembre de 2003,884 . Ello representa un incremento mayor a la población de adultos privados de la libertad. 
El $62 \%$ de estos adolescentes procede de familias incompletas y desestructuradas, donde la figura paterna está comúnmente ausente. Su condición expresa una marcada desintegración familiar, por la cual no es posible garantizar mecanismos de control de su propio comportamiento, como consecuencia de figuras parentales y de autoridad ausentes, así como de adultos que monitoreen y proporcionen retroinformación apropiada sobre el desempeño social de estos adolescentes desde edades tempranas (Defensoría del Pueblo, 2000).

Además de estas características es importante señalar que muchos de ellos consumen drogas (inhalantes como Terokal, Marihuana y Pasta Básica de Cocaína: PBC), tienen un manejo inadecuado de su sexualidad que los lleva a ser padres prematuramente y carecen de documentos personales que afectan el ejercicio de sus derechos generales y específicos (partida de nacimiento, boleta militar, entre otros), de acuerdo con los resultados de este estudio.

Asimismo, presentan dificultades para reconocer y respetar derechos y normas sociales establecidas, y carecen de habilidades psicológicas básicas para controlar sus impulsos y conductas violentas y depresivas (Defensoría del Pueblo, 2000).

Otro aspecto directamente relacionado con las modalidades de atención del Poder Judicial hacia los adolescentes en conflicto con la Ley Penal, consiste en el motivo de ingreso a los centros juveniles. De acuerdo con las estadísticas disponibles, la mayoría de delitos o infracciones corresponden al robo, en sus modalidades de robo simple y agravado. Este grupo de delitos se asocia directamente con los niveles de pobreza presentes en la gran mayoría de adolescentes procesados y sentenciados, y obedece a tipos delictivos poco o nada violentos, salvo los casos cuyo agravante es indicativo del uso de la violencia física. 
Sin embargo, los otros grupos de delitos como violación, homicidios, lesiones graves y leves, cuya proporción estadística es menor, son clasificados como delitos violentos y están estrechamente relacionados a patrones de conducta antisocial precoces en su aparición y persistentes en su duración temporal. Por otro lado, aunque no se incluye en la siguiente figura, debe mencionarse que algunos adolescentes aplican a más de un tipo de delito, y su reincidencia es considerablemente baja (ver Figuras 4 y 5).
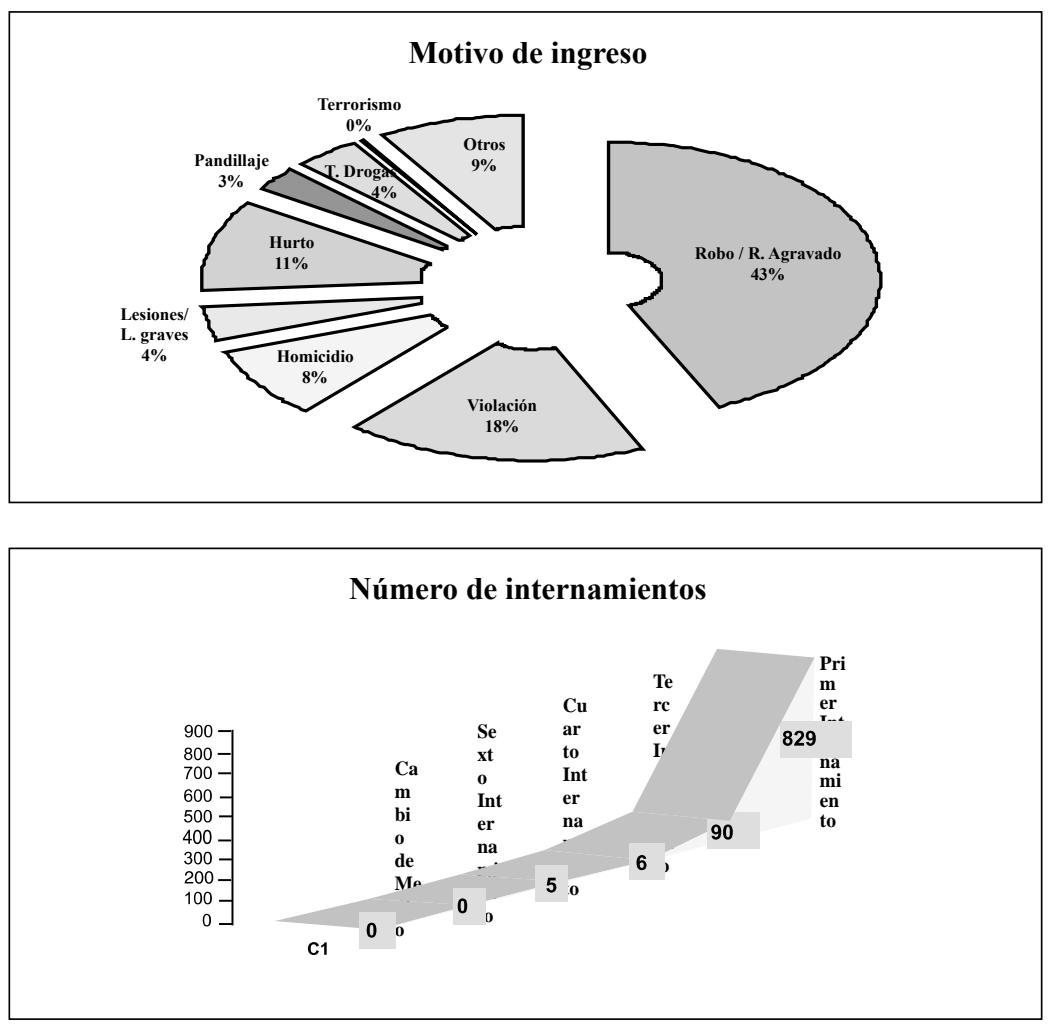

Figuras 4 y 5. Fuente y elaboración Gerencia de Centros Juveniles del Poder Judicial del Perú (2004). 
Respecto a la edad, se observa una tendencia natural a concentrar mayor prevalencia de delitos en los grupos de edad comprendidos entre los 15 y 18 años. Asimismo, se observa que conforme los adolescentes de la población en tutela judicial ascienden en edad, disminuye la prevalencia de casos delictivos. Este fenómeno ha sido reportado por Moffitt (1993a) como "la historia natural del delito" y representa una evidencia empírica y transcultural sólida respecto a la prevalencia de dos tipos de patrones de conducta delictiva durante la edad adolescente, que será explicado más adelante. Por otro lado, debe indicarse que esta distribución "espontánea" de las edades no se vincula con la sensibilidad o mecanismos de filtro del Sistema Penal Juvenil al momento de detectar a los adolescentes con comportamiento antisocial (ver Figura 6). Pues sea, a través de una mayor o menor identificación policial de estos adolescentes, las edades tenderían a mantener una distribución similar de acuerdo con esta teoría.

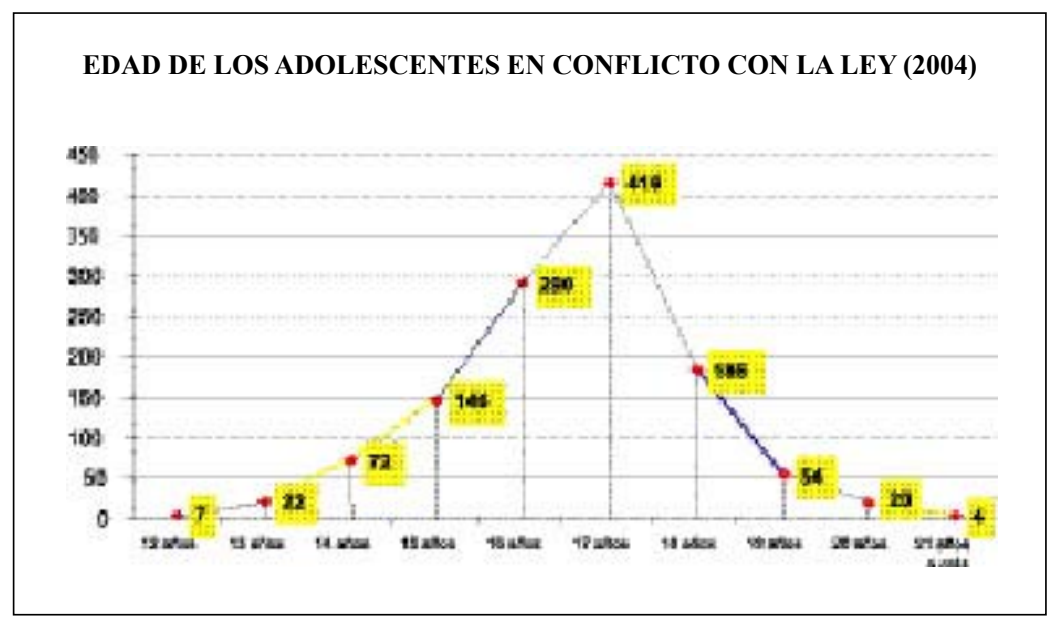

Figura 6. Fuente y elaboración Gerencia de Centros Juveniles del Poder Judicial del Perú (2004). 


\section{Los Centros Juveniles en el Perú}

Los Centros Juveniles en Perú, desde la creación del primer Correccional de Menores en 1902, han sido administrados por diversas instituciones como la Iglesia Católica, la Policía Nacional, el Ministerio de Justicia, el Ministerio de Salud, el Ministerio de Educación y el Ministerio de la Presidencia a través del Instituto Nacional de Bienestar Familiar (INABIF). Mediante el Decreto Legislativo $N^{\circ}$ 866, Ley de Organización y Funciones del Ministerio de la Mujer y el Desarrollo Humano, de fecha 25 de octubre de 1996, se transfiere a la Secretaría Ejecutiva de la Comisión Ejecutiva del Poder Judicial las funciones relacionadas con la rehabilitación para la reinserción en la sociedad de los adolescentes infractores de la Ley Penal, es decir, aquellos que cumplen una medida socioeducativa en libertad o privativa de tal, con lo cual el Poder Judicial del Perú asumió la labor de atención y rehabilitación de estos adolescentes.

Para este propósito, se crea la Gerencia de Operaciones de Centros Juveniles como órgano de línea de la Gerencia General de la Secretaría Ejecutiva de la Comisión Ejecutiva del Poder Judicial, el 25 de noviembre de 1996, hoy Gerencia de Centros Juveniles, órgano de línea de la Gerencia General del Poder Judicial. Desde ese entonces, la Gerencia de Centros Juveniles tiene el encargo de ofrecer atención integral a estos adolescentes, coherente con las normas internacionales y nacionales vigentes sobre administración de justicia a menores de edad, como la Constitución Política del Estado y el Código de los Niños y Adolescentes de Perú, tendientes a la promoción y mantenimiento de la paz con justicia social. En este contexto se desarrollaron una serie de estrategias de intervención con miras a elevar la calidad del servicio y brindar una posibilidad de cambio basándose en un trabajo técnico planificado en beneficio de esta población. 
La Gerencia de Centros Juveniles tiene como principal objetivo institucional y compromiso social rehabilitar al adolescente infractor favoreciendo, de esta manera, su reinserción social efectiva. Para ello se elaboró y se aprobó mediante Resolución Administrativa del Titular del Pliego del Poder Judicial No 539-97, del 25 de noviembre de 1997, el Sistema de Reinserción Social del Adolescente Infractor y su modificatoria aprobada por R.A. N ${ }^{\circ}$ 075-SE-TP-CME-PJ del 03 de febrero del 2000.

\section{El Sistema de Reinserción Social del Adolescente Infractor en el Perú}

Se trata de un sistema de atención integral especializado en el tratamiento del adolescente infractor, el cual comprende una serie de programas, métodos, técnicas e instrumentos de carácter eminentemente educativos, acorde con las leyes y normas compatibles con los derechos humanos, en el ámbito nacional e internacional (Reglas de Beijing, Directrices de Riyadh y Reglas para la protección de jóvenes privados de su libertad, instrumentos propuestos por las Naciones Unidas).

En este sistema, el adolescente infractor es visto como una persona en desarrollo, sujeto de derechos y protección, quien debido a múltiples causas ha cometido una infracción a la ley, por tanto, requiere de atención especial e individualizada que permita desarrollar sus potencialidades, habilidades, valores y hábitos adecuados, dentro de un proceso educativo integral. Dicho proceso se lleva acabo a través del medio abierto y el medio cerrado, según la medida socioeducativa judicial que corresponda.

El Nuevo Código de los Niños y Adolescentes de Perú (Ley 27337), establece que el internamiento preventivo de los adolescentes infractores de la Ley Penal, así como la medida socioeducativa 
de internamiento se cumplen en los Centros Juveniles del Poder Judicial. El ingreso a los centros, así como su permanencia en ellos, se establece a través de una Sentencia Judicial. Este mismo principio rige para las medidas socioeducativas no privativas de la libertad.

El sistema de Justicia Penal Juvenil del Perú garantiza el derecho del adolescente a preservar su identidad y privacidad, más aun cuando se encuentra en situación de víctima, autor, partícipe o testigo de una infracción, falta o delito, prohibiéndose conforme a ley en todos estos casos el conocimiento público de su identidad a través de los medios de comunicación.

\section{Etiología de la delincuencia juvenil I: los factores asociados}

Angenent y De Mann (1996) definen la conducta antisocial de tipo delincuencial en jóvenes como aquellas actividades que en términos de las normas y costumbres se consideran indeseables o incluso inaceptables. Las formas más graves se llaman trastornos de conducta, por lo que los autores concluyen que la delincuencia juvenil es un trastorno del comportamiento penado por la ley.

En lo concerniente a factores externos asociados al comportamiento antisocial en adolescentes, se ha aludido con frecuencia a los valores de la comunidad o del entorno del mesosistema (Bronfenbrenner, 1999). Asimismo, el tipo de vecindario en que viven los adolescentes desde temprana infancia y el estrato socioeconómico de procedencia son buenos predictores del comportamiento antisocial (Frías-Armenta et al., 2003). Entre los factores interpersonales debemos mencionar tres especialmente importantes: familia, escuela y grupos de pares.

Con respecto a la familia, se han identificado factores estructurales como el tamaño de la familia, el trabajo de las madres, el 
orden de nacimiento de los hijos y la ausencia de uno de los progenitores (especialmente la figura paterna). Posteriormente se prestó mayor atención a factores dinámicos tales como el clima familiar, la calidad de las relaciones vinculares, el apego del adolescente hacia sus padres, la comunicación intrafamiliar, los estilos de crianza y la disciplina del hogar.

La supervisión y el monitoreo de los padres parece ser un factor muy significativo, especialmente en el caso de los adolescentes varones (Angenent \& De Mann, 1996). Asimismo, Farrington et al. (2001) encuentran una alta concentración de delincuentes en las familias, por ejemplo, el arresto de un familiar, particularmente del padre o de alguno de la misma generación, incrementa la probabilidad de que algún miembro de la familia de la siguiente generación (hijos, sobrinos, nietos) sea delincuente.

Acerca de la escuela, las experiencias de fracaso escolar constituyen con frecuencia un factor de riesgo mientras que el logro escolar representa un factor protector. También resultan importantes la actitud del adolescente hacia la escuela -es decir, si la considera un espacio placentero y útil para su desarrollo personaly el compromiso con las metas de aprendizaje. En cuanto al grupo de pares, frecuentar amigos que son delincuentes, portan armas (blancas o de fuego) o consumen drogas, constituyen un buen predictor de la delincuencia juvenil (Seydlitz \& Jenkins, 1998). Al respecto, Killias y Ribeaud (1999), en un estudio realizado en 12 países de Europa y Estados Unidos, encuentran una alta relación entre el consumo de drogas y los delitos contra la propiedad y el tráfico de drogas, aunque esto por sí solo no predice la delincuencia entre adolescentes.

Con respecto a los factores internos o intrapersonales, además de la edad, el género y las experiencias normativas de vida, se encuentran los aspectos biológicos, cognitivos y afectivos. Los fac- 
tores biológicos incluyen la herencia, especialmente en los casos en que existe algún trastorno de personalidad asociado. También ha sido estudiado el efecto de las hormonas -principalmente los efectos de la testosterona- durante las etapas pre natal y puberal, así como los efectos de los bajos niveles de serotonina en el cerebro.

Henry y Moffitt (1992), utilizando técnicas de neuroimagen, encontraron correlatos neurológicos de deficiencias ejecutivas en muestras de adolescentes delincuentes precoces. Éstas incluyeron déficits en habilidades neuropsicológicas como comprensión verbal, atención, concentración, formación de conceptos, abstracción, anticipación y planificación. Del mismo modo, un bajo nivel intelectual parece contribuir al riesgo de cometer delitos.

En lo que respecta a los factores afectivos, se ha estudiado de manera especial la relación existente entre psicopatología y delito. La asociación más evidente tiene que ver con el trastorno antisocial de la personalidad y sus precursores en la infancia: trastorno de déficit de atención por hiperactividad, trastorno oposicionista y trastorno de conducta (Lahey \& Loeber, 1992). Asimismo, han sido identificados algunos rasgos de personalidad frecuentes entre infractores como son la impulsividad, dificultad para postergar la gratificación, autoconcepto disminuido, falta de habilidades sociales, bajo nivel de empatía y poca capacidad para sentir culpa (Blackburn, 1995).

De acuerdo con la clasificación de Lykken (2000), en el espectro del delito perpetrado por adolescentes, un grupo de adolescentes infractores y delincuentes juveniles delinquen como consecuencia de tres factores predisponentes que pueden constituirse progresivamente en un patrón de comportamiento antisocial:

1. Intensificación de las transformaciones psicológicas propias del periodo evolutivo adolescente. 
2. Exposición temprana a una socialización deficiente como consecuencia de una práctica familiar negligente y composición familiar insuficiente, lo cual daría origen a la Sociopatía.

3. Presencia de rasgos temperamentales elevados como la búsqueda de sensaciones, la impulsividad y la ausencia de miedo, que desencadenarían la Psicopatía.

Como se ha podido revisar hasta el momento, las características individuales y del entorno resultan importantes predictores de la delincuencia juvenil. Sin embargo, pese a existir consenso respecto a una participación simultánea entre variables externas e internas, son estas últimas las que generan mayor controversia. Al respecto, Quay (1987) indica que en casi la mayor parte de los casos, los delitos violentos y el crimen se asocian más con factores internos y con una mayor perturbación psicológica en comparación con delitos cometidos por adolescentes que constituyen faltas menores hacia la autoridad parental y no parental.

En todo caso, un patrón persistente de episodios de delitos perpetrados antes y durante la adolescencia constituye el mejor criterio predictivo para clasificar entre grupos de adolescentes en riesgo de convertirse en futuros delincuentes adultos, y adolescentes que experimentan una intensificación de algunas de las características de su personalidad durante este periodo evolutivo.

Al respecto, es importante indicar que, en ambos grupos de adolescentes, aquello que llamamos "delito" representa un espectro de comportamientos antisociales que están tipificados penalmente por la legislación judicial penal de un país (Tiffer, 2003). En ese sentido, existen formas de violencia ejercidas por adolescentes y jóvenes que no están tipificadas como delitos (Howe, 1997). Del mismo modo, existen determinados tipos de delitos que no son violentos en su perpetración (como sustraer dinero o tarjetas bancarias de otras personas sin que éstas lo sepan), 
pese a que podríamos decir que todo delito "en sí mismo", es un tipo de violencia contra las personas (Quay, 1987).

A continuación presentamos la adaptación de un modelo empírico para los problemas de conducta durante la adolescencia propuesto por Dodge \& Pettit (2003), que resume de manera esquemática las vías explicativas causales, moderadoras y mediadoras del comportamiento de infracción a la Ley Penal en esta población (ver Figura 7).

Modelo biopsicosocial del desarrollo del comportamiento de infracción a la Ley durante la adolescencia

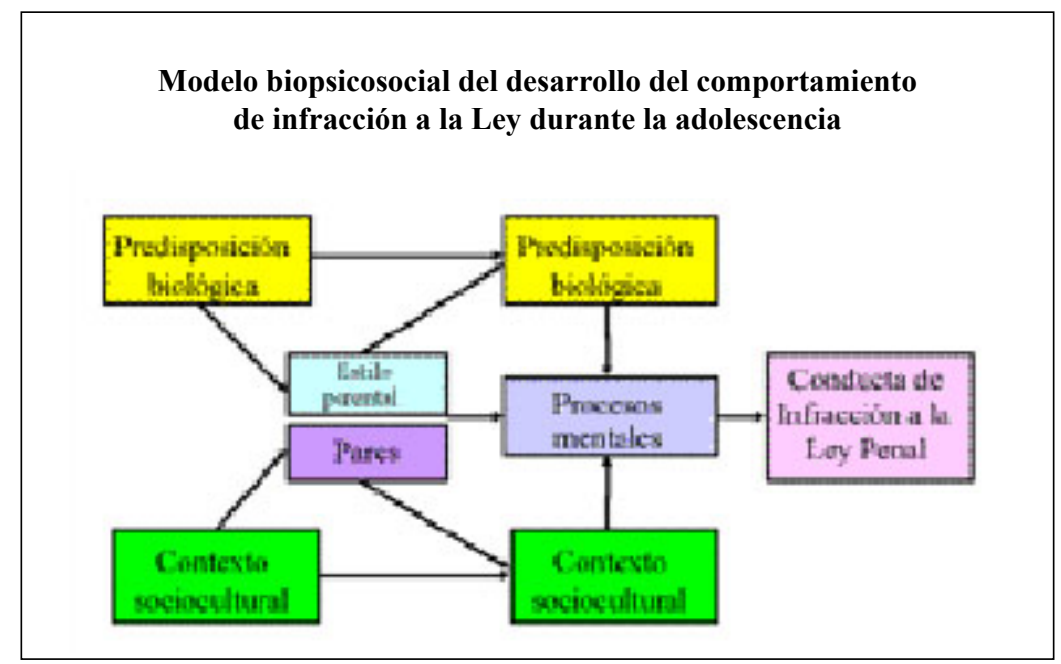

Figura 7. Adaptación propuesta por los autores al Modelo de Dodge y Pettit (2003).

Por otro lado, no podemos considerar el problema de la violencia adolescente y juvenil como un fenómeno aislado de otros comportamientos problemáticos y de otros factores de riesgo psicosocial (Caspi, Henry, McGee, Moffitt \& Silva, 1995; Henry, Caspi, Moffitt, Harrington \& Silva, 1999; Henry, Feehan, McGee, 
Stanton, Moffitt \& Silva, 1993; Killias \& Ribeaud, 1999; Moffitt, 1993a; OMS, 2003).

Al respecto, la investigación ha demostrado que no todos los adolescentes y jóvenes violentos son iguales. Existen adolescentes y jóvenes violentos que, pese a su funcionamiento psicológico violento, no comenten delitos a pesar de estar expuestos al riesgo de perpetrarlos (Lykken, 2000). Contrariamente, existen adolescentes y jóvenes que han cometido delitos sin que necesariamente presenten este tipo de funcionamiento psicológico (Iza, 2002; Moffitt, 1993 ${ }^{\text {, }}$ 1993b).

Los adolescentes y jóvenes violentos tienden a cometer una variedad de delitos, además de presentar adicionalmente una variedad de problemas conductuales asociados a su comportamiento delictivo; entre ellos se encuentran un alto ausentismo o deserción escolar, abuso de sustancias psicoactivas, características personales tales como impulsividad y oposicionismo intensificadas, mentiras compulsivas, y altas tasas de enfermedades de transmisión sexual (Caspi, Moffitt, Silva, Stouthamer-Loeber, Schmutte \& Krueger, 1994; Jaffee, Moffitt, Caspi, Taylor \& Arseneault, 2002; Koenen, Moffitt, Caspi, Taylor \& Purcell, 2003; Krueger, Schmutte, Caspi, Moffitt, Campbell \& Silva, 1994).

No obstante, es importante destacar que no todos los adolescentes y jóvenes con la totalidad o alguno de los problemas conductuales mencionados serán necesariamente violentos o delincuentes; asimismo, no todos los adolescentes y jóvenes delincuentes presentan consistentemente estos problemas (Broidy, Nagin, Tremblay, Brame, Dodge, Fergusson, Horwood, Loeber, Laird, Lynam \& Moffitt, 2003; Iza, 2002; Morales, 2004).

Sin embargo, aquellos adolescentes y jóvenes que desde la niñez y la pubertad han sido expuestos a una serie de desventajas a 
lo largo de su desarrollo tales como cuidados negligentes, pobre estimulación temprana (Henry, Moffitt, Robins, Earls \& Silva, 1993), aprovisionamiento insuficiente, y que además reúnen una serie de déficits neuropsicológicos verbales y ejecutivos, acompañados de desórdenes severos del desarrollo, como déficit atencional e hiperactividad (Henry, Caspi, Moffitt \& Silva, 1996), tienen mayor probabilidad de desarrollar un patrón de conducta antisocial persistente a lo largo del ciclo de vida (Baltes, Lindenberger \& Staudinger, 1997; Caspi, McClay, Moffitt, Mill, Martin, Craig, Taylor \& Poulton, 2002; Caspi \& Roberts, 2001; Lahey \& Loeber, 1992; OMS, 2003).

Al respecto, debe señalarse que no ocurre lo mismo con sus pares adolescentes, que de modo casi independiente de los entornos criminógenos en que se hayan desarrollado (Bronfenbrenner, 1999; Bronfenbrenner \& Ceci, 1994; Bronfenbrenner \& Morris 1997), no presentan las mismas características de desventaja personal y no presentan, por tanto, el mismo patrón de conducta (Moffitt, 1993a, 1996).

Si este último grupo de adolescentes cometiera algún tipo de delito, su conducta antisocial tendría que ser explicada directa y principalmente por los efectos de los entornos ambientales en los que interactúan (Frías-Armenta, López- Escobar \& Díaz-Méndez, 2003) y por los procesos de socialización negligentes sobre los que se han desarrollado (Blunt, Bugental \& Goodnow, 1997); no necesariamente por variables individuales. Ello permite indicar que dicho comportamiento antisocial-delictivo se presenta de manera limitada al periodo de la adolescencia (Moffitt, 1993b, 2001, 2002).

Entre los factores de vulnerabilidad identificados más importantes, la investigación ha demostrado que los adolescentes de género masculino tienen una mayor probabilidad de pertenecer al 
grupo de adolescentes que muestra comportamiento antisocial persistente a lo largo de la vida, en comparación con sus respectivos pares femeninos (Magdol, Moffitt, Caspi, Newman, Fagan \& Silva, 1997; Moffitt, Caspi, Rutter \& Silva, 2001).

Otro importante grupo de factores de riesgo identificados en esta población lo constituye su asociación con otros tipos de violencia. Presenciar actos violentos en el hogar o sufrir abuso físico o sexual puede condicionar a los niños y adolescentes a considerar la agresión como un medio aceptable para resolver problemas o interactuar con los demás (Jaffee, Moffitt, Caspi, Taylor \& Arseneault, 2002; Koenen, Moffitt, Caspi, Taylor \& Purcell, 2003; OMS, 2003).

Del mismo modo, la exposición prolongada a conflictos armados como el terrorismo también puede contribuir a sostener una cultura del terror que haga más fácil la aparición de adolescentes y jóvenes violentos (Bandura, 1977). En ese sentido, la comprensión de los factores que incrementan el riesgo de que los adolescentes y jóvenes se conviertan en víctimas y perpetradores de actos violentos, como delitos y crímenes, es esencial para formular políticas y programas eficaces de prevención de la violencia adolescente y juvenil (OMS, 2003; Peñaherrera, 1998).

Cabe mencionar que el delito no es un constructo psicológico sino una categoría jurídico-legal bajo la cual no es posible agrupar a todos los delincuentes existentes, pues éstos son muy diferentes entre sí, y el único elemento común a todos ellos es la conducta o el acto mismo de delinquir. Este acto reúne un conjunto de variables psicológicas organizadas consistentemente, configurando un patrón de conducta al cual los psicólogos denominan comportamiento antisocial (Farrington, 1983; Iza, 2002).

En este sentido, es preciso indicar que las correspondencias entre los dominios del delito y del comportamiento antisocial son 
unidireccionales, es decir, todo delito representa un tipo de comportamiento antisocial (Blackburn, 1995), pero no todo comportamiento antisocial constituye un delito, en tanto no haya sido tipificado como tal en la legislación penal de una Nación (Iza, 2002). Por lo que debe señalarse que en la legislación penal peruana, los adolescentes que delinquen no son llamados delincuentes, sino infractores. La razón obedece a que la trasgresión a la Ley Penal por parte de un adolescente en el Perú es considerada una infracción a la Ley.

\section{Etiología de la delincuencia juvenil II: las trayectorias del de- sarrollo}

En el campo de la violencia y la criminalidad, el término trayectoria se ha utilizado para referirse a la evolución o curso de un comportamiento cuando no se recibe tratamiento o intervención alguna. En la salud pública se emplea el término "historia natural de una enfermedad" para referirse a esta idea.

Estudiar la violencia criminal como la posibilidad de una trayectoria en el comportamiento delincuencial es un paso muy importante para el entendimiento del problema y el diseño de políticas públicas de prevención de la violencia criminal focalizada especialmente en población joven. Implica que se está considerando que la violencia y el crimen no son sólo producto de las circunstancias del momento como la falta de vigilancia, un conflicto interpersonal, el consumo abusivo de alcohol, o la tenencia de armas, sino también producto de una historia personal del neurodesarrollo social. Por qué y cuándo se inicia, cuáles son los signos tempranos y cómo evoluciona, son elementos indispensables para pensar en prevenir o interrumpir esta historia.

Asimismo, es importante saber si existe más de una trayectoria, pues ello podría implicar la existencia de diferentes tipos del 
problema con distintas causas, cursos y pronósticos; y consecuentemente, diferentes formas de prevenir o de tratar. Muchas de las teorías predominantes al interior de la Criminología moderna tienden a englobar a la población de violentos o de infractores como si fuera una población homogénea, explicando las diferencias en la edad de aparición, persistencia o severidad del crimen como distintos niveles del mismo problema (Bandura, 1973; Gottfredson \& Hirschi, 1990; Sutherland \& Cressey, 1999). A continuación revisaremos algunas de las más importantes contribuciones al respecto.

\section{Según el tipo de delito: especialización vs. versatilidad}

Una forma frecuente de clasificar a los delincuentes es por el tipo de delito. La evidencia empírica sobre la tendencia a cometer un sólo tipo de delito (especialización) versus la versatilidad en la actividad delincuencial es controversial.

Por un lado, algunos investigadores han encontrado que sí existe esta tendencia (Farrington, Snyder \& Finnegan, 1988), en especial para aquellos que continúan delinquiendo en la edad adulta y durante más tiempo (Blumstein, Cohen, Das \& Moitra, 1988), aquellos involucrados en delitos de "cuello blanco" (Benson \& Moore, 1992), y los que inician actividades delincuenciales luego de tener problemas de adicción a drogas (Farabee, Joshi \& Anglin, 2001).

Incluso, sobre la base de estudios de gemelos y estudios de adopción, Mednick y Kandel (1998) piensan que posiblemente existen factores genéticos que predisponen hacia los delitos contra la propiedad mientras que factores perinatales parecen ser importantes para los delitos contra las personas. 
No obstante la evidencia anterior, luego de una revisión exhaustiva de la literatura sobre el tema cubriendo los años de 1966 a 1995, Simon (1997) concluyó que si bien existen algunos delincuentes especializados en algunos tipos de delitos, la mayoría, en especial los más persistentes, no tienden a especializarse. Investigaciones posteriores a esta revisión brindan soporte adicional a esta conclusión (Piquero, 2000; Piquero \& Buka, 2002).

Estudios sobre las trayectorias del comportamiento criminal por tipo de delito muestran diferencias en la frecuencia de la actividad delincuencial con picos alrededor de los 16 años de edad, sin importar el tipo de delito (Brame, Mulvey \& Piquero, 2001). Igualmente, para aquellos que son persistentes en su conducta antisocial, los dos grupos (especializados y no especializados) no parecen diferir en sus antecedentes familiares o personales (Farrington, 1989).

En 1998, Rolf Loeber y su grupo de investigación en la Universidad de Pittsburg propusieron, basándose en un seguimiento longitudinal durante 14 años a una cohorte de niños $(\mathrm{n}=517)$, tres trayectorias para el desarrollo del comportamiento criminal (Loeber, Farrington, Stouthamer-Loeber, Moffitt \& Caspi, 1998).

En las tres trayectorias, los comportamientos más serios son precedidos por otros menos graves. Un primer camino, denominado abierto o público, se inicia con una agresión menor (molestar o amedrentar a otros), seguido por participar en peleas con enfrentamientos físicos y verbales, terminando en delitos violentos.

Un segundo camino es denominado vía encubierta y se inicia con comportamientos encubiertos menores como decir mentiras, robar pertenencias de otros u objetos en venta de las tiendas, progresa a daños a la propiedad, y termina en delitos menores a serios como fraude, robo y asalto. 
El tercer camino se inicia a través del comportamiento desafiante y oposicionista, progresa a desobediencia y termina en desacatamiento de normas de funcionamiento familiar y social, como escaparse de la casa o la escuela, o permanecer fuera del hogar por muchas horas y hasta tarde.

Soporte empírico adicional para este modelo surge de los análisis de los datos de la National Youth Survey (una encuesta nacional tomada a una muestra aleatoria de jóvenes de ambos sexos entre 12 y 17 años de edad en los EE.UU.) y del Chicago Youth Development Study (un estudio de seguimiento de niños de quinto y séptimo grado de escuelas públicas en la ciudad de Chicago).

En esta prueba del modelo, el $84 \%$ de los delincuentes cumplieron los pasos o caminos propuestos por Loeber et al. (Tolan \& Gorman-Smith, 1998). Este porcentaje fue mayor cuando se limitó el análisis al subgrupo de delincuentes más serios o violentos.

\section{Según el patrón de agresión: agresión reactiva vs. proactiva}

Dodge (1991) ha propuesto la existencia de dos tipos de conductas agresivas: una agresión reactiva y otra proactiva, aunque es frecuente observar los dos tipos en un mismo individuo. La primera es un tipo de agresión que se produce en reacción a la provocación, de allí el nombre de reactiva.

Teóricamente, estas personas no iniciarían peleas pero serían muy sensibles a cierto tipo de estímulos (cuestionamientos a su identidad o poder, ofensas a la autoestima) y reaccionan con ira en forma descontrolada y desproporcionada. En cambio, la agresión proactiva se utiliza para obtener algún bien o beneficio (objetos o dominación de otro, por lo que tiene un carácter eminentemente 
instrumental) y suele carecer de manifestaciones de afecto (se trata de una acción fría y calculada).

Los dos tipos de agresión corresponden a diferentes estructuras, conexiones, circuitos y sistemas de neurotransmisión a nivel cerebral (según lo observado en modelos animales), diferentes procesos cognoscitivos, y probablemente diferentes etiologías. Dodge (1991) propuso, a manera de hipótesis, que la agresión reactiva podría ser el resultado de experiencias en la infancia y la niñez que disminuyen la sensación de seguridad y elevan los niveles de estrés como, por ejemplo, la pérdida de un ser querido y las amenazas crónicas (el maltrato y el abuso durante la niñez o el ser testigo de violencia), especialmente cuando son impredecibles. En cambio, la agresión proactiva podría ser el resultado de una alta exposición y valoración de respuestas agresivas (en la familia, la comunidad o la televisión) y una falta de exposición a comportamientos prosociales (modelos de conducta socialmente adecuados).

La investigación empírica sobre estos dos patrones conductuales de agresión es limitada. Sin embargo, se ha encontrado que la agresión reactiva se asocia más frecuentemente con antecedentes de maltrato y estrategias disciplinarias severas y aparece a más temprana edad (alrededor de los 4 años de edad).

Los niños con este patrón de agresión manifiestan hipersensibilidad y tendencia a malinterpretar signos sociales, generan rechazo entre sus pares y maestros (Dodge, Lockman, Harnish, Bates \& Pettit, 1997), y tienen mayor riesgo de agredir a su pareja (Brengden, Vitaro, Tremblay \& Lavoie, 2001).

Por el contrario, la agresión proactiva aparece alrededor de los 6 años de edad (Dodge et al., 1997) y predice conductas delincuenciales durante la adolescencia (Brengden et al., 2001; 
Vitaro, Gendreau, Tremblay \& Oligny, 1998), explicadas especialmente por los efectos del aprendizaje social durante la socialización infantil.

Establecer las diferencias entre estos dos tipos de agresión y sus factores determinantes tiene importantes implicancias para la prevención y el control de la violencia y la criminalidad. Primero, porque es muy probable que ciertos programas tengan mejores resultados con algún tipo de agresión que con otro. En segundo lugar, porque conociendo los factores determinantes de cada tipo de agresión, es posible diseñar programas de prevención primaria. Por ejemplo, ciertos factores como el alcohol y el estrés podrían ser más importantes para los reactivos que para los proactivos. La evolución de cada tipo de agresión nos podría también orientar sobre los momentos apropiados de la intervención.

\section{Según la edad de iniciación y persistencia: precoces vs. tardíos}

En una publicación de 1989, Patterson, DeBaryshe y Ramsey plantearon la existencia de al menos dos caminos a la delincuencia o criminalidad: uno de iniciación en la edad escolar y otro de inicio en la adolescencia. Según estos autores, unas prácticas de crianza inapropiadas serían el factor que conduciría a la aparición del problema en ambos casos.

En este mismo sentido, Moffitt (1993a) también propuso la existencia de dos grupos de adolescentes antisociales: 1) limitados a la adolescencia (adolescence-limited) y 2) persistentes a través de la vida (life-course-persistent). Según sus investigaciones, estos últimos, correspondientes a la minoría dentro de la población de delincuentes, se caracterizan por la aparición temprana (incluso desde la edad preescolar) y persistente de un conjunto de problemas de comportamiento que irían escalando en 
frecuencia y severidad y, si bien cambian en sus manifestaciones según la edad, correspondía al mismo tipo de problema (continuidad heterotípica). Por ejemplo, la agresión en la edad preescolar podría manifestarse como rabietas, en la edad escolar como destructividad y agresión hacia otros en la adolescencia.

Por el contrario, Moffitt (1993a) postula que los autolimitados a la adolescencia corresponden a la gran mayoría de jóvenes que alguna vez se han involucrado en actividades delincuenciales y se distinguen porque carecen de problemas de conducta notorios durante su niñez.

La confluencia de estos dos grupos explicaría por qué se observan tasas de participación en delincuencia y violencia especialmente altas durante la adolescencia. La desaparición del grupo de autolimitados explicaría el descenso que se observa en estas tasas luego de la adolescencia. El soporte empírico para esta taxonomía es aún incipiente pero persuasivo (Bartusch, Lynam, Moffitt \& Silva, 1997; Chung, Hill, Hawkins, Gilchrist \& Nagin, 2002; Moffitt, 1993a; Moffitt \& Caspi, 2001; Moffitt, Caspi, Harrington \& Milne, 2002; Nagin, Farrington \& Moffitt, 1995; Simons, Wu, Conger \& Lorenz, 1994; Tolan \& Thomas, 1995; Vitelli, 1997).

Los estudios mencionados incluyen poblaciones de Canadá, Inglaterra, Nueva Zelanda, Suecia y EE.UU. También existen estudios colombianos demostrando la existencia de estos dos grupos aunque aún restringidos a población de delincuentes adultos (Klevens, Restrepo, Roca \& Martínez, 2000; Klevens \& Roca, 1999). Estos dos caminos parecen ser similares entre hombres y mujeres (Moffitt \& Caspi, 2001), aunque algunos estudios son consistentes en señalar un mejor ajuste del modelo a muestras de adolescentes varones. 
Además de la diferencia en la edad de inicio del comportamiento antisocial, se encuentran diferencias en sus factores determinantes. Los factores asociados al camino precoz y persistente son múltiples e incluyen: problemas neurocognitivos (hiperactividad, problemas de atención, impulsividad, bajo nivel de habilidades verbales), rasgos de personalidad (temperamento difícil, reactividad emocional negativa, tendencia a ser temerario y a buscar lo novedoso), prácticas de crianza inapropiadas (estrategias disciplinarias agresivas e inconsistentes, carencia de interacción interpersonal positiva, falta de supervisión) y conflicto familiar (Bartusch et al., 1997; Klevens, Restrepo, Roca \& Martínez, 2000; Moffitt et al., 2001; Moffitt, Caspi, Harrington \& Milne, 2002; Simons et al., 1994).

En contraste, el grupo de inicio tardío tiene pocos factores de riesgo, es decir, es bastante parecido a la población de jóvenes que no se involucra en hechos delictivos excepto por dos características: mayor frecuencia de interrupciones en la supervisión adulta y mayor tiempo de exposición a pares antisociales (Bartusch et al., 1997; Klevens et al., 2000; Patterson \& Yoerger, 1997; Simons et al., 1994).

Los dos grupos difieren también en su pronóstico. Para los precoces y persistentes, el pronóstico es bastante reservado. Además de los riesgos de criminalidad y violencia, tienen mayores probabilidades de fracaso y deserción escolar, consumo temprano y excesivo de alcohol y drogas, precocidad y promiscuidad sexual, infracción de normas de tránsito, inestabilidad laboral y afectiva, y violencia doméstica (Farrington, 1995; Klevens et al., 2000).

Por el contrario, el grupo tardío tiende a involucrarse en delitos de menor gravedad (delitos contra la propiedad, los de "cuello blanco", y los relacionados con el narcotráfico; Loeber, 1990). Sin 
embargo, aún se carece de suficiente evidencia sobre su eventual desistencia, y aún cuando desisten, parecen tener otros problemas como beber más, usar drogas con más frecuencia e involucrarse en peleas (Nagin, Farrington \& Moffitt, 1995).

Otros investigadores han encontrado más de dos caminos: precoces y persistentes de alta actividad versus precoces y persistentes con baja actividad sin diferencias en sus factores determinantes (Nagin et al., 1995). En otro estudio, se describe un grupo de precoces que, excepto por la edad de iniciación, se parecen a los tardíos, es decir, se involucran en delitos de menos seriedad y desisten al llegar a la edad adulta (Chung et al., 2002). El factor que diferencia a este grupo de los precoces y persistentes es que viven en barrios donde la exposición a pares antisociales y la disponibilidad de drogas es menor.

Una de las limitaciones de las dos clasificaciones anteriores es que mezclan violencia con otros comportamientos antisociales o criminales como fugarse de la casa, robo y consumo de drogas. Tremblay y sus colaboradores han realizado varios estudios en Montreal documentando la historia natural de la agresión física. Primero, basándose en observaciones de niños y reportes de sus madres, obtuvieron evidencia de que el comportamiento agresivo aparece en algunos niños desde los 7 meses de edad. El porcentaje de niños mostrando ese comportamiento se incrementa en la medida en que ganan movilidad, de manera que antes de los 2 años de edad, la mayoría de los niños han sido alguna vez físicamente agresivos con otros (Tremblay, Japel, Pérusse, Boivan, Zoccolillo, Montplaisir et al., 1999).

Esto parece ser el pico para el comportamiento agresivo, porque según los datos de un estudio transversal en una muestra representativa de niños canadienses, el porcentaje de niños con 
comportamientos agresivos disminuye progresivamente a partir de los 3 años de edad (Morales, 2004).

En otro estudio con una cohorte de niños seguidos desde los 6 hasta los 15 años de edad, Nagin y Tremblay (1999) encontraron que alrededor de $5 \%$ de su cohorte mostraba comportamiento agresivo persistente, mientras que $20 \%$ a $30 \%$ de los niños tenían altos niveles de agresión a los 6 años, y otro 50\% tenía niveles moderados de agresión pero desistía con el tiempo. Alrededor de la mitad del grupo con agresión persistente manifestaba también comportamiento oposicionista.

Finalmente se constató que este grupo de agresores persistentes fue el que se involucró con mayor frecuencia en delitos violentos durante su adolescencia. Este grupo de agresores persistentes parece corresponder al grupo que hemos llamado precoces $\mathrm{y}$ persistentes, y aunque Nagin y Tremblay rechazan la idea de un grupo de aparición tardío, en otra publicación describen trayectorias de niños que no manifiestan comportamientos agresivos a los 6 años pero muestran comportamientos violentos de manera transitoria o con baja frecuencia (Brame, Nagin \& Tremblay, 2001), lo cual concuerda con la descripción de tardíos propuestos por Moffitt (1993a).

\section{Conclusiones y recomendaciones}

Existe consenso respecto a la estrategia para prevenir la violencia juvenil. Se considera que, junto a otros comportamientos de riesgo, el fortalecimiento de políticas públicas eficaces y sostenidas representa la mejor alternativa para promover la calidad de vida, el desarrollo y la inserción social de los adolescentes y jóvenes (Cortázar, Francke \& La Rosa, 1998; Francke, 1998; La Rosa, 1998). Por lo tanto, es necesario replantear y reformular las estrategias actuales 
para atender y prevenir el comportamiento antisocial durante la adolescencia, a la luz de las evidencias provenientes de la investigación.

La existencia de una trayectoria de iniciación precoz de comportamientos antisociales, con altos niveles de persistencia y múltiples consecuencias negativas en la edad adulta, tiene importantes implicancias para el desarrollo de políticas públicas de prevención del crimen juvenil, así como el tratamiento jurídico y psicológico por parte de los sistemas de administración de Justicia Penal Juvenil en nuestras naciones (Morales, 2005b).

Aunque el grupo de adolescentes precoces y persistentes corresponde a la minoría dentro de la población de delincuentes, pareciera generar aproximadamente el $50 \%$ de la totalidad de los delitos denunciados y no denunciados en el mundo (Farrington, 1995; Tracy, Wolfgang \& Figlio, 1990). En nuestro medio no existen estudios que asocien los perfiles de riesgo del comportamiento antisocial adolescente y sus correlatos criminales en records policiales; debido a ello, no es posible afirmar que existe la presencia de infractores juveniles persistentes o no persistentes involucrados en tasas altas o bajas de incidentes violentos.

Pese a la ausencia de registros claros de las características de los infractores juveniles, la existencia de una población adulta-joven equivalente al $10 \%$ del total de una de las penitenciarías de adultos más grandes del país ${ }^{4}$, que estuvo en algún momento de su vida en un Centro Juvenil, permitiría sospechar que en nuestro medio podría estar presente un patrón persistente de comportamiento antisocial que se remonta a la adolescencia.

4 Penitenciaría para varones de Lurigancho en Lima, Perú, cuya población superó los ocho mil internos durante el 2004 (L. Francia, Comisionado del Programa de Asuntos Penales y Penitenciarios de la Defensoría de Pueblo del Perú, comunicación personal). 
Existen algunos esfuerzos de investigación que tienen el propósito de identificar la existencia de este patrón entre la población de adolescentes en conflicto con la Ley Penal atendidos por la Gerencia de Centros Juveniles del Poder Judicial peruano. De comprobarse la presencia de perfiles de riesgo de comportamiento antisocial persistente y no persistente, se tendría evidencia para implementar un sistema de atención y prevención del comportamiento antisocial entre esta población; podrían considerarse, para ello, intervenciones focalizadas y especializadas de acuerdo a cada perfil de riesgo. Una lógica de intervenciones en esta línea representa una adecuada alternativa para los servicios públicos de atención y prevención de la violencia juvenil por múltiples razones. En primer término, porque cualquier tipo de intervención psicológica debe estar basada en algún tipo de evidencia sobre su eficacia, de manera que pueda prestarse un servicio de atención apropiado que garantice los resultados esperados. En segundo término, porque los recursos para el financiamiento de los servicios de atención a esta población no son abundantes y, por lo tanto, existe una responsabilidad técnica y moral de destinar los fondos disponibles sobre aquellos programas de intervención que han demostrado algún nivel significativo de resultados, o que se diseñan sobre la base de algún fundamento teórico pertinente, y a la vez permiten medir los resultados preestablecidos.

El valor de utilizar el conocimiento psicológico para atender los problemas de conducta y prevenir episodios de mayor severidad resulta evidente cuando, además de observar los resultados de los análisis costo-beneficio de los programas basados en evidencia, se observa la reducción de otros problemas asociados (comórbidos), comúnmente consecuentes con la conducta delictiva, como el consumo abusivo de alcohol y drogas (Morales, 2005a).

Resulta indispensable destacar que la prevención del comportamiento antisocial adolescente no debería orientarse exclusivamente 
hacia la atención y la rehabilitación del daño una vez producido, es decir, destinar los esfuerzos de intervención, inclusive aquellos sumamente especializados y demostrados científicamente como eficaces, cuando la conducta antisocial o el problema de conducta (su precursor) se han originado y se manifiestan bajo múltiples formas de funcionamiento personal y en diversos entornos de desarrollo.

De acuerdo a lo anterior se deriva la necesidad de una intervención muy temprana, idealmente desde el embarazo, promoviendo los determinantes biopsicosociales de la salud del niño durante su desarrollo temprano. Estas intervenciones buscarían reducir complicaciones de embarazo y parto, y mejorar las estrategias de crianza, especialmente durante los primeros años de vida.

Las imágenes observadas documentando el impacto del maltrato y la deprivación psicoafectiva en las estructuras y fisiología cerebrales durante el periodo en que estas estructuras están aún en desarrollo (primeros tres años; Teicher, 2002) sugieren la urgencia de actuar en este sentido. Desafortunadamente, los programas de prevención existentes se inician en la edad escolar cuando ya el problema de comportamiento es evidente y, aunque muestran impacto, son programas de muy alto costo (ver por ejemplo, Conduct Problems Prevention Research Group, 1999).

Existen programas de prevención de la violencia juvenil de éxito científicamente comprobado como, por ejemplo, el programa reportado por Berrueta-Clement, Schweinhart, Barnett y Weikart en 1987. Este programa brindó cuidados y una educación preescolar de alta calidad, que redujo la incidencia de delincuencia juvenil a la cuarta parte y criminalidad adulta a la cuarta parte, además de logros muy positivos en escolaridad y empleo, con un balance muy favorable en términos de costo-beneficio (Karoly, Greenwood, Everingham et al., 1998) que, sin embargo, no ha sido implementado a gran escala. 
En el caso de los adolescentes antisociales tardíos (limitados a la adolescencia), Moffitt (1993a) propone, a manera de hipótesis, que éstos se involucran en actividades delincuenciales por satisfacer necesidades comunes a los adolescentes de la población general: aceptación del grupo, status, independencia, diversión, entre otras necesidades normativas de la edad. Desafortunadamente en muchos países de América Latina, particularmente en el Perú, la jornada escolar en la escuela pública es corta y muchos adolescentes carecen de supervisión entre las horas de salida de la escuela y la llegada de los padres del trabajo, mientras las oportunidades para involucrarse en problemas con la Ley están siempre presentes.

Para el grupo de adolescentes en riesgo, se piensa que los programas de prevención deberían crear y mantener permanentemente actividades con supervisión adulta en donde los adolescentes tengan oportunidad para observar e interactuar con personas que les sirvan de ejemplo o modelamiento positivo. En este sentido, no parecen estar mal encaminados aquellos programas como las casas o clubes juveniles, grupos de teatro y organizaciones deportivas. La experiencia de la "Casa de la Juventud" en el distrito de Surquillo en la ciudad de Lima, así como los programas de desarrollo juvenil de CEDRO y los de algunos municipios, representan extraordinarios esfuerzos locales que deberían ser replicados al interior del país.

Finalmente, si bien el conocimiento de trayectorias parece complicar el control del problema de la violencia adolescente y juvenil, también amplía sustancialmente las posibilidades de su prevención oportuna y eficaz. 


\section{Referencias}

American Psychiatric Association. (1994). Diagnostic and statistical manual of mental disorders (4a. ed.). Washington, DC: Masson.

Angenent, H. \& De Mann, A. (1996). Background factors of juvenil delinquency. Nueva York: Peter Lang.

APOYO Opinión y Mercado. (2003). Informe gerencial de marketing. Perfil del adolescente y joven. Lima: Autor.

Baltes, P. B., Lindenberger, U. \& Staudinger, U. M. (1997). Lifespan theory in developmental psychology. En W. Damon \& R. Lerner (Eds.), Handbook of child psychology ( $5^{\mathrm{a}}$. ed., Cap. 18). Nueva York: John Wiley \& Sons.

Bandura, A. (1973). Aggression: A social learning analysis. Englewood Cliffs, NJ: Prentice Hall.

Bandura, A. (1977). Social learning theory. Englewood Cliffs, NJ: Prentice Hall.

Barletta, M. C. \& Morales, H. (2004, junio). Lineamientos para una Política Criminal acorde a la Doctrina de la Protección Integral. Infancia, Boletín del Instituto Interamericano del Niño, 73, 240.

Bartusch, D. R., Lynam, D. R., Moffitt, T. E. \& Silva, P. A. (1997). Is age important? Testing a general versus a developmental theory of antisocial behavior. Criminology, 35, 13-47.

Benson, M. L. \& Moore, E. (1992). Are white collar and common offenders the same? An empirical and theoretical critique of a recently proposed general theory of crime. Journal of Research in Crime and Delinquency, 29, 251-272.

Berrueta-Clement, J. R., Schweinhart, L. J., Barnett, W. S. \& Weikart, D. P. (1987). The effects of early educational intervention on crime and delinquency in adolescence and early adulthood. En J. D. Burchard \& S. N. Burchard (Eds.), Primary prevention of psychopathology: Vol. 10. Prevention of delinquent behavior. Newbury Park, CA: Sage. 
Blackburn, R. (1995). The psychology of criminal conduct: Theory, research and practice. Chichester, Reino Unido: John Wiley and Sons.

Blascovich, J. (2000). Using physiological indexes of psychological processes in social psychological research. En H. T. Reis \& C. M. Judd (Eds.), Handbook of research methods in social and personality psychology. Nueva York: Cambridge University Press.

Blumstein, A., Cohen, J., Das, S. \& Moitra, S. D. (1988). Specialization and seriousness during adult criminal careers. Journal of Quantitative Criminology, 4, 303-345.

Blunt, L., Bugental, D. \& Goodnow, J. (1997). Socialization processes. En W. Damon \& R. Lerner (Eds.), Handbook of child psychology (5a. ed., Cap. 7). Nueva York: John Wiley \& Sons.

Brame, R., Mulvey, E. P. \& Piquero, A. R. (2001). On the development of different kinds of criminal activity. Sociological Methods \& Research, 29, 319-341.

Brame, B., Nagin, D. S. \& Tremblay, R. E. (2001). Developmental trajectories of physical aggression from school entry to late adolescence. Journal of Child Psychology and Psychiatry, 42, 503-512.

Brengden, M., Vitaro, F., Tremblay, R. E. \& Lavoie, F. (2001). Reactive and proactive aggression: Predictors to physical violence in different contexts and moderating effects of parental monitoring and caregiving behavior. Journal of Abnormal Child Psychology, 29, 293-304.

Broidy, L. M., Nagin, D. S., Tremblay, R. E., Brame, B., Dodge, K., Fergusson, D. et al. (2003). Developmental trajectories of childhood disruptive behavior disorders and adolescent delinquency: A six-nation replication. Developmental Psychology, 39, 222-245.

Bronfenbrenner, U. (1999). Environments in developmental perspective: Theoretical and operational models. En S. L. Friedman (Ed.), Measuring environment across the life span: Emerging methods 
and concepts. Washington, DC: American Psychological Association.

Bronfenbrenner, U. \& Ceci, S. (1994). Nature-nurture reconceptualized in developmental perspective: A bioecological model. Psychological Review, 101, 4, 568-586.

Bronfenbrenner, U. \& Morris, P. (1997). The ecology of developmental processes. En W. Damon \& R. Lerner (Eds.), Handbook of child psychology (5a. ed., Cap. 17). Nueva York: John Wiley \& Sons.

Caspi, A., Henry, B., McGee, R. O., Moffitt, T. E. \& Silva, P. A. (1995). Temperamental origins of child and adolescent behavior problems: From age 3 to age 15. Child Development, 66, 55-68.

Caspi, A., McClay, J., Moffitt, T. E., Mill, J., Martin, J., Craig, I. W. et al. (2002). Role of genotype in the cycle of violence in maltreated children. Science, 297, 851-854.

Caspi, A., Moffitt, T. E., Silva, P. A., Stouthamer-Loeber, M., Schmutte, P. \& Krueger, R. (1994). Are some people crimeprone? Replications of the personality-crime relation across nation, gender, race, and method. Criminology, 32, 301-333.

Caspi, A. \& Roberts, B. W. (2001). Personality development across the life course: The argument for change and continuity. Psychological Inquiry, 12, 49-66.

Centro de Estudios y Acción para la Paz. (2000). Foro nacional: el sistema de justicia penal juvenil en el Perú, análisis y propuestas. Lima: Defensoría del Pueblo-Comunidad Europea.

Chung, I. J., Hill, K. G., Hawkins, J. D., Gilchrist, L. D. \& Nagin, D. S. (2002). Childhood predictors of offense trajectories. Journal of Research in Crime and Delinquency, 39, 60-90.

Conduct Problems Prevention Research Group. (1999). Initial impact of the fast track prevention trial for conduct problems: I. The high risk sample. Journal of Consulting and Clinical Psychology, 67, 631-647. 
Cortázar, J., Francke, P. \& La Rosa, L. (1998). Políticas sociales para la adolescencia y juventud en el Perú. Socialismo y Participación, 81, 9-35.

Defensoría del Pueblo. (2000). El Sistema Penal Juvenil en el Perú (Informe No. 51). Lima: ILANUD-Comisión Europea.

Dirección de Familia y Participación Ciudadana de la Policía Nacional del Perú. (2004). Diagnóstico situacional de la violencia juvenil en Lima y Callao. Lima: Autor.

Dodge, K. A. (1991). The structure and function of reactive and proactive aggression. En D. J. Peplar \& K. H. Rubin (Eds.), The development and treatment of childhood aggression (pp. 210-212). Hillsdale, NJ: Lawrence Erlbaum.

Dodge, K. A., Lochman, J. E., Harnish, J. D., Bates, J. E. \& Pettit, G. S. (1997). Reactive and proactive aggression in school children and psychiatrically impaired chronically assaultive children. Journal of Abnormal Psychology, 106, 1, 37-51.

Dodge, K. A. \& Pettit, G. S. (2003). A biopsychosocial model of the development of chronic conduct problems in adolescence. Developmental Psychology, 39, 2, 349-371.

Eley, T. C., Lichtenstein, P. \& Moffitt, T. E. (2003). A longitudinal behavioral genetic analysis of the etiology of aggressive and non-aggressive antisocial behavior. Development and Psychopathology, 15, 2, 383-402.

Erikson, E. (1980). Identidad, juventud y crisis. Madrid: Taurus.

Farabee, D., Joshi, V. y Anglin, M. D. (2001). Addiction careers and criminal specialization. Crime and Delinquency, 47, 196220.

Farrington, D. P. (1983). Epidemiology. En H. Quay (Ed.), Handbook of juvenil delinquency. Nueva York: John Wiley \& Sons.

Farrington, D. P. (1989). Early predictors of adolescent aggression and adult violence. Violence and Victims, 4, 79-100. 
Farrington, D. P. (1995). The Twelfth Jack Tizard Lecture. The development of offending and antisocial behavior from childhood: Key findings from the Cambridge Study in Delinquent Development. Journal of Child Psychology and Psychiatry, 360, 929-964.

Farrington, D. P., Jollife, D., Loeber, R., Stouthamer-Loeber, M. \& Kalb, L. (2001). The concentration of offenders in families, and family criminality in the prediction of boy's delinquency. Journal of Adolescence, 24, 579-596.

Farrington, D. P., Snyder, H. \& Finnegan, T. (1988). Specialization in juvenile court careers. Criminology, 26, 461-488.

Francke, P. (1998). Pobreza y juventud. Socialismo y Participación, $81,37-58$.

Frías-Armenta, M., López-Escobar, A. E. \& Díaz-Méndez, S. G. (2003). Predictores de la conducta antisocial juvenil: un modelo ecológico. Estudios de Psicología, 8, 1, 15-24.

Garrido, V. (1997). Principios de criminología. Valencia: Torant le Blanch.

Gottfredson, M. R. \& Hirschi, T. (1990). A general theory of crime. Stanford: Stanford University Press.

Henry, B., Caspi, A., Moffitt, T. E., Harrington, H. \& Silva, P. A. (1999). Staying in school protects boys with poor selfregulation in childhood from later crime: A longitudinal study. International Journal of Behavioral Development, 23, 1049-1073.

Henry, B., Caspi, A., Moffitt, T. E. \& Silva, P. A. (1996). Temperamental and familial predictors of violent and non-violent criminal convictions: From age 3 to age 18. Developmental Psychology, 32, 614-623.

Henry, B., Feehan, M., McGee, R., Stanton, W., Moffitt, T. E. \& Silva, P. A. (1993). The importance of conduct problems and depressive symptoms in predicting adolescent substance use. Journal of Abnormal Child Psychology, 21, 469-480. 
Henry, B. \& Moffitt, T. E. (1992). Neuropsychological and neuroimaging studies of juvenil delinquency and adult criminal behavior. En D. Stoff, J. Breiling \& J. Maser (Eds.), Handbook of antisocial behavior. Nueva York: John Wiley \& Sons.

Henry, B., Moffitt, T. E., Robins, L. N., Earls, F. \& Silva, P. A. (1993). Early family predictors of child and adolescent antisocial behavior: Who are the mothers of delinquents? Criminal Behavior and Mental Health, 3, 97-118.

Hirschi, T. (1990). Causes of delinquency. Berkeley, CA: University of California Press.

Howe, J. (1997). Juvenil justice and youth violence. Thousand Oaks CA: Sage.

Instituto Nacional de Estadística e Informática. (1998). Estado de la población peruana 1998: situación de la población joven y de la tercera edad. Lima: Autor.

Instituto Nacional de Estadística e Informática. (2004). Compendio estadístico 2004. Lima: Autor.

Iza, M. (2002). Personalidad, adolescencia y delito. Un estudio en adolescentes institucionalizados de Lima-Perú. Tesis de maestría, Universidad de Salamanca, España.

Jaffee, S. R., Moffitt, T. E., Caspi, A., Taylor, A. \& Arseneault, L. (2002). Influence of adult domestic violence on children's internalizing and externalizing problems. An environmentally informative twin study. Journal of the American Academy of Child and Adolescent Psychiatry, 41, 1095-1103.

Jaffee, S. R., Moffitt, T. E., Caspi, A. \& Taylor, A. (2003). Life with (or without) father. The benefits of living with two biological parents depend on the father's antisocial behavior. Child Development, 74, 109-126.

Jeglum-Bartusch, D. R., Lynam, D. R., Moffitt, T. E. \& Silva, P. A. (1997). Is age important? Testing general versus developmental theories of antisocial behavior. Criminology, $35,13-47$. 
Karoly, L. A., Greenwood, P. W., Everingham, S. S., Houbé, J., Kilburn, M. R., Rydell, C. P. et al. (1998). Investing in our children: What we know and don't know about the costs and benefits of early child interventions. Santa Monica, CA: Rand.

Kazdin, A. \& Buela-Casal, G. (2001). Conducta antisocial. Evaluación, tratamiento y prevención en la infancia y la adolescencia. Madrid: Pirámide.

Killias, M. \& Ribeaud, D. (1999). Drug use and crime among juveniles. An international perspective. Studies on Crime and Crime Prevention, 8, 2, 189-205.

Klevens, J., Restrepo, O., Roca, J. \& Martínez, A. (2000). Comparison of offenders with early and late-starting antisocial behavior in Colombia. International Journal of Offender Therapy and Comparative Criminology, 44, 195204.

Klevens, J. \& Roca, J. (1999). Nonviolent youth in a violent society: Resilience and vulnerability in the country of Colombia. Violence and Victims, 14, 311-322.

Koenen, K. C., Moffitt, T. E., Caspi, A., Taylor, A. \& Purcell, S. (2003). Domestic violence is associated with environmental suppression of IQ in young children. Development \& Psychopathology, 15, 297-311.

Krueger, R. F., Schmutte, P. S., Caspi, A., Moffitt, T. E., Campbell, K. \& Silva, P. A. (1994). Personality traits are linked to crime among males and females: Evidence from a birth cohort. Journal of Abnormal Psychology, 103, 328-338.

Lahey, B. \& Loeber, R. (1992). Attention-deficit/hiperactivity disorder, oppositional defiant disorder, conduct disorder, and adult antisocial behavior: A life span perspective. En D. Stoff, J. Breiling \& J. Maser (Eds.), Handbook of antisocial behavior. Nueva York: John Wiley \& Sons.

Lahey, B., Moffitt, T. E. \& Caspi, A. (Eds.). (2003). The causes of conduct disorder and serious juvenile delinquency. Nueva York: Guilford. 
La Rosa, L. (1998). Institucionalidad pública de políticas sociales para adolescentes y jóvenes. Socialismo y Participación, 81, 59-83.

Lerner, R. M. \& Galambos, N. L. (1998). Adolescent development: Challenges and opportunities for research, programs, and policies. Annual Review of Psychology, 49, 413-446.

Loeber, R. (1990). Development and risk factors of juvenile antisocial behavior and delinquency. Clinical Psychology, 10, $1-41$.

Loeber, R., Farrington, D. P., Stouthamer-Loeber, M., Moffitt, T. E. \& Caspi, A. (1998). The development of male offending: Key findings from the first decade of the Pittsburgh Youth Study. Studies on Crime and Prevention, 7, 141-171.

Lykken, D. (2000). Las personalidades antisociales. Barcelona: Herder.

Lynam, D., Caspi, A., Moffitt, T. E., Wikstrom, P., Loeber, R. \& Novak, S. (2000). The interaction between impulsivity and neighborhood context on offending: The effects of impulsivity are stronger in poorer neighborhoods. Journal of Abnormal Psychology, 109, 563-574.

Magdol, L., Moffitt, T. E., Caspi, A., Newman, D. L., Fagan, J. \& Silva, P. A. (1997). Gender differences in partner violence in a birth cohort of 21-year-olds: Bridging the gap between clinical and epidemiological approaches. Journal of Consulting and Clinical Psychology, 65, 68-78.

Mednick, S. A. \& Kandel, E. S. (1998). Congenital determinants of violence. Bulletin of the American Academy of Psychiatry and the Law, 16, 101-109.

Mill, J. S., Caspi, A., McClay, J., Sugden, K., Purcell, S., Asherson, P. et al. (2002). The dopamine D4 receptor and the hyperactivity phenotype: A developmental-epidemiological study. Molecular Psychiatry, 7, 383-391.

Moffitt, T. E. (1993a). Adolescence-limited and life-coursepersistent antisocial behavior: A developmental taxonomy. Psychological Review, 100, 4, 674-701. 
Moffitt, T. E. (1993b). The neuropsychology of conduct disorder. En J. E. Richters \& D. Cicchetti (Eds.), Developmental perspectives on conduct disorder. Development and Psychopathology, 5, 135151.

Moffitt, T. E. (1996). Editorial: A primer on measuring children's antisocial behaviors. Journal of the American Medical Association, 275, 5, 403-404.

Moffitt, T. E. (en prensa). Quantitive behavioural genetic research into human anti-social behaviour: 1929-2001. Genetics and Human Behaviour.

Moffitt, T. E., Brammer, G. L., Caspi, A., Fawcett, J. P., Raleigh, M., Yuwiler, A. Et al. (1998). Whole blood serotonin relates to violence in an epidemiological study. Biological Psychiatry, 43, 446-457.

Moffitt, T. E. \& Caspi, A. (1998). Annotation: Implications of violence between intimate partners for child psychologists and psychiatrists. Journal of Child Psychology and Psychiatry, 39, 2, 137-144.

Moffitt, T. E. \& Caspi, A. (2001). Childhood predictors differentiate life-course persistent and adolescence-limited antisocial pathways among males and females. Development \& Psychopathology, 13, 355-375.

Moffitt, T. E., Caspi, A., Dickson, N., Silva, P. A. \& Stanton, W. (1996). Childhood-onset versus adolescent-onset antisocial conduct in males: Natural history from age 3 to 18. Development \& Psychopathology, 8, 399-424.

Moffitt, T. E., Caspi, A., Harrington, H. \& Milne, B. J. (2002). Males on the life-course-persistent and adolescence-limited antisocial pathways. Follow-up at age 26 years. Development \& Psychopathology, 14, 179-207.

Moffitt, T. E., Caspi, A., Rutter, M. \& Silva, P. A. (2001). Sex differences in antisocial behavior: Conduct disorder, delinquency, and violence in the Dunedin Longitudinal Study. Cambridge, Reino Unido: Cambridge University Press. 
Moffitt, T. E., Krueger, R. F., Caspi, A. \& Fagan, J. (2000). Partner abuse and general crime: How are they the same? How are they different? Criminology, 38, 201-235.

Moffitt, T. E., Lynam, D. R. \& Silva, P. A. (1994). Neuropsychological tests predict persistent male delinquency. Criminology, 32, 101124.

Moffitt, T. E., Robins, R. W. \& Caspi, A. (2001). A couple's analysis of partner abuse with implications for abuse prevention. Criminology and Public Policy, 1, 401-432.

Morales, H. (2004, junio). El adolescente infractor en conflicto con la ley penal: una perspectiva socio-psicológica del sistema de justicia penal juvenil en el Perú. Psicólogo Interamericano, Boletín de la Sociedad Interamericana de Psicología, 84.

Morales, H. (2005a). Consumo de alcohol y sustancias psicoactivas entre adolescentes en conflicto con la Ley Penal. Un análisis descriptivo. Revista Peruana de Drogodependencias, 3.

Morales, H. (2005b, abril). Estado del arte en el estudio del desarrollo del comportamiento criminal durante la adolescencia: implicancias para el diseño de políticas eficaces de prevención de la delincuencia juvenil y la administración de justicia penal Juvenil. Ponencia presentada en el 11vo. Congreso de las Naciones Unidas en Prevención y Justicia Criminal, Bangkok, Tailandia.

Nagin, D. S., Farrington, D. P. \& Moffitt, T. E. (1995). Life-course trajectories of different types of offenders. Criminology, 33, 111-139.

Nagin, D. S. \& Tremblay, R. E. (1999). Trajectories of boys' physical aggression, opposition, and hyperactivity on the path to physically violent and nonviolent juvenile delinquency. Child Development, 70, 1181-1196.

Organización Mundial de la Salud. (2003). Informe mundial sobre la violencia y la salud. Washington, DC: Autor. 
Patterson G. R., DeBaryshe, B. D. \& Ramsey, E. A. (1989). Developmental perspective on antisocial behavior. American Psychologist, 44, 329-335.

Patterson, G. R. \& Yoerger, K. (1997). A developmental model for late-onset delinquency. En D. W. Osgood (Ed.), Motivation and delinquency (Vol. 44, pp. 119-177). Lincoln, NE: University of Nebraska Press.

Pattishall, E. (1994). A research agenda for adolescent problems and risk-taking behaviors. En R. Ketterlinus \& M. Lamb (Eds.), Adolescent problem behaviors: Issues and research. Nueva Jersey: Lawrence Erlbaum.

Peñaherrera, E. (1998). Comportamiento de riesgo adolescente: una aproximación psicosocial. Revista de Psicología de la PUCP, 16, 2, pp.

Piquero, A. (2000). Frequency, specialization, and violence in offending careers. Journal of Crime and Delinquency, 37, 392-418.

Piquero, A. R. \& Buka, S. L. (2002). Linking juvenile and adult patterns of criminal activity in the Providence cohort of the National Collaborative Perinatal Project. Journal of Criminal Justice, 30, 259-272.

Quay, H. (1987). Patterns of delinquent behavior. En H. Quay, Handbook of juvenile delinquency. Nueva York: John Wiley $\&$ Sons.

Seydlitz, R. \& Jenkins, P. (1998). The influence of family, friends, schools, and community on delinquent behavior. En T. Gullota, G. Adams \& R. Montemayor (Eds.), Delinquent violent youth. Trends and interventions. Thousand Oaks, CA: Sage.

Simon, L. M. (1997). Do criminal offenders specialize in crime types? Applied and Preventive Psychology, 6, 35-53.

Simons, R. L., Wu, C., Conger, R. D. \& Lorenz, F. O. (1994). Two routes to delinquency: Differences between early and late starters in the impact of parenting and deviant peers. Criminology, 32, 247-275. 
Sutherland, E. H. \& Cressey, D. R. (1999). A theory of differential association. En F. T. Cullen \& R. Agnew (Eds.), Criminological theory: Past to present (pp. 82-84). Los Angeles, CA: Roxbury.

Teicher, M. (2002). Scars that won't heal: The neurobiology of child abuse. Scientific American, 286, 68-75.

Tiffer, C. (2003, noviembre). Justicia Penal Juvenil. Instrumentos internacionales de Naciones Unidas y la experiencia de Costa Rica. Entre la Protección Integral y el Derecho Penal Juvenil. Ponencia presentada en el Primer Congreso Mundial sobre Derechos de la Niñez y la Adolescencia, Estado de Nueva Esparta, Venezuela.

Tolan, P. H. \& Gorman-Smith, D. (1998). Development of serious and violent offending. En R. Loeber \& D. P. Farrington (Eds.), Serious and violent offenders: Risk factors and successfuliInterventions (pp. 77-83). Thousand Oaks, CA: Sage.

Tolan, P. H. \& Thomas, T. (1995). The implications of age of onset for delinquency risk II: Longitudinal data. Journal of Abnormal Child Psychology, 23, 157-181.

Tracy, P. E., Wolfgang, M. E. \& Figlio, R. M. (1990). Delinquency careers in two birth cohorts. Nueva York: Plenum Press.

Tremblay, R. E., Japel, C., Pérusse, D., Boivan, M., Zoccolillo, M., Montplaisir, J. et al. (1999). The search for age of "onset" of physical aggression: Rousseau and Bandura revisited. Criminal Behavior and Mental Health, 9, 8-23.

Vitaro, F., Gendreau, P. L., Tremblay, R. E. \& Oligny, P. (1998). Reactive and proactive aggression differentially predict later conduct problems. Journal of Child Psychology and Psychiatry, 39, 377-385.

Vitelli, R. (1997). Comparison of early and late start models of delinquency in adult offenders. International Journal of Offender Therapy and Comparative Criminology, 41, 351357. 
Wright, B. R. E., Caspi, A., Moffitt, T. E. \& Silva, P. A. (1999a). Reconsidering the relationship between SES and delinquency: Causation but not correlation. Criminology, 37, 175-194.

Wright, B. R. E., Caspi, A., Moffitt, T. E. \& Silva, P. A. (1999b). Low self-control, social bonds, and crime: Social causation, social selection, or both? Criminology, 37, 479-514.

Wright, B. R. E., Caspi, A., Moffitt, T. E. \& Silva, P. A. (2001). The effects of social ties on crime vary by criminal propensity: A life-course model of interdependence. Criminology, 39, 321-351. 